\title{
Miniband Transport in a Two-dimensional Electron Gas with a Strong Periodic Unidirectional Potential Modulation
}

\author{
S. K. Lyo ${ }^{\dagger}$ and W. Pan* \\ ${ }^{\dagger}$ Department of Physics and Astronomy, University of California, Irvine, CA, 92697 \\ ${ }^{*}$ Sandia National Laboratories, Albuquerque, NM 87185, USA
}

\begin{abstract}
We study the Bloch oscillations of a two-dimensional electron gas with a strong periodic potential-modulation and miniband transport along the field at low temperatures, assuming a free motion in the transverse direction. The dependence of the current on the field, the electron density, and the temperature is investigated by using a relaxation-time approximation for inelastic scattering. For a fixed total scattering rate, the field dependence of the current is sensitive to the ratio of the elastic and inelastic scattering rates in contrast with the recent result of a multiband but otherwise similar model with a weak potential modulation.

Keywords: A. Semiconductors; C. Two-dimensional systems: D. High-field transport
\end{abstract}

Typeset using REVTEX

1

(C) 2014. This manuscript version is made available under the Elsevier user license http://www.elsevier.com/open-access/userlicense/1.0/ 
An electron undergoes a periodic motion in a strong electric field $F$ in a periodic lattice with a frequency $f=p e F / 2 \pi \hbar \equiv \omega_{F} / 2 \pi$, where $\omega_{F}$ is the angular speed and $p$ is the lattice period. ${ }^{1}$ In a typical lattice with a short lattice period, these so-called Bloch oscillations are damped out quickly because of the short scattering time $\tau$ before the electrons sweep through the band (i.e., $\omega_{F} \tau \ll 1$ ). In a superlattice with a large lattice period, however, the Bloch frequency can fall in a terahertz region, yielding $\omega_{F} \tau \gtrsim 1$ under a moderate field, ${ }^{2}$ yielding Bloch oscillations with a negative differential conductance in the steady-state. ${ }^{3-5}$

This interesting phenomenon has received much attention in the past for its potential to yield terahertz generation, detection, and also for applications to negative differential conductance. Past experimental ${ }^{6-8}$ and theoretical ${ }^{3,9-11}$ studies $^{12}$ have mainly been focused on three-dimensional (3D) vertical quantum-well (QW) superlattice structures ${ }^{3}$ for technical feasibility and one-dimensional (1D) superlattices ${ }^{4,12}$ for theoretical simplicity, while very few studies have been carried out in two-dimensional (2D) systems. Theoretical results of miniband transport in 1D and 3D systems seem to yield roughly similar qualitative behavior, although the interesting prediction that the frequency of the Bloch oscillation depends only on the elastic scattering rate was obtained only in a $1 \mathrm{D}$ analytic result. ${ }^{4}$

Recently, 2D systems with patterned surface structures have been studied for various advantages. ${ }^{13}$ The $I-V$ data were explained by employing a Kronig-Penney model for a $2 \mathrm{D}$ electron gas (2DEG) with a weak 1D potential modulation along the field direction. ${ }^{5}$ It was found there that, for a fixed total scattering rate, the $I-V$ curve is nearly independent of the ratio $R$ of the inelastic and elastic scattering rates with a relaxation time approximation for the latter. It was speculated there that this result arises from the combination of the 2D system and weak potential modulation, because this behavior is in contrast to that in strongly modulated 1D and 3D miniband systems, where the $I-V$ curve is sensitive to $R^{4,3}$ In this paper, we investigate miniband transport in a $2 \mathrm{DEG}$ produced by a strong potential modulation along the field. We find that the $I-V$ curve is sensitive to $R$ in this system just as in 1D and 3D minibands. We also study the peak current and the field at the peak as a function of the electron density and show that these quantities are sensitive to $R$. 
The steady-state Boltzmann equation is given, in a relaxation-time approximation, ${ }^{3,4}$ by

$$
-\frac{e F}{\hbar} \frac{\partial f(\mathbf{k})}{\partial k_{x}}=-\nu_{i n}\left[f(\mathbf{k})-f^{(0)}\left(\varepsilon_{\mathbf{k}}\right)\right]-\frac{2}{\tilde{\rho}} \sum_{\mathbf{k}^{\prime}}\left[f(\mathbf{k})-f\left(\mathbf{k}^{\prime}\right)\right] \delta\left(\varepsilon_{\mathbf{k}}-\varepsilon_{\mathbf{k}^{\prime}}\right)
$$

where $\nu_{\text {in }}$ is the inelastic scattering rate assumed to be a constant, $\mathbf{k}$ is the wave vector, $f^{(0)}(\varepsilon)$ is the Fermi function, $f(\mathbf{k})$ is the nonequilibrium distribution function, and $\tilde{\rho}=$ $\hbar / \pi\left|U_{\mathbf{k}, \mathbf{k}^{\prime}}\right|^{2}$. The intraband elastic scattering matrix element $U_{\mathbf{k}, \mathbf{k}^{\prime}}$ is approximated as a constant assuming a short-range potential. The energy is given by $\varepsilon_{\mathbf{k}} \equiv \varepsilon\left(k_{x}, k_{y}\right)=\varepsilon_{y}\left(k_{y}\right)+$ $\varepsilon_{x}\left(k_{x}\right)$, where $\varepsilon_{x}(x)=\Delta(1-\cos x) / 2, \varepsilon_{y}\left(k_{y}\right)=\hbar^{2} k_{y}^{2} / 2 m^{*}, m^{*}$ is the effective mass and $-\pi \leq x \equiv p k_{x} \leq \pi$. The bandwidth $\Delta$ is generated by tunneling between the adjacent deep wells produced by the potential-energy modulation in the $x$ direction parallel to the field $F$.

Defining $\nu(\varepsilon)=\nu_{\text {in }}+\nu_{e l}(\varepsilon), \nu_{e l}(\varepsilon)=\rho(\varepsilon) / \tilde{\rho}$, where $\rho(\varepsilon)$ is the density of states and $h(\varepsilon)=(2 / \tilde{\rho}) \sum_{\mathbf{k}^{\prime}} f\left(\mathbf{k}^{\prime}\right) \delta\left(\varepsilon-\varepsilon_{\mathbf{k}^{\prime}}\right)$ we recast Eq. (1) into $^{3}$

$$
-\frac{p e F}{\hbar} \frac{\partial f(\mathbf{k})}{\partial x}=-\nu\left(\varepsilon_{\mathbf{k}}\right) f(\mathbf{k})+\nu_{i n} f^{(0)}\left(\varepsilon_{\mathbf{k}}\right)+h\left(\varepsilon_{\mathbf{k}}\right)
$$

Note that Eq. (2) conserves the number of particles. Defining

$$
f(\mathbf{k})=\phi(\mathbf{k}) \exp \left[\frac{\hbar}{p e F} \int_{0}^{x} \nu\left(\varepsilon\left(k_{x}^{\prime}, k_{y}\right)\right) d x^{\prime}\right]
$$

$\gamma_{i n}=p e F / \hbar \nu_{i n}$, and inserting in Eq. (2), we find

$$
\phi(\mathbf{k})=\int_{x}^{\infty} \tilde{G}\left(\varepsilon\left(k_{x}^{\prime}, k_{y}\right)\right) \exp \left[-\frac{\hbar}{p e F} \int_{0}^{x^{\prime}} \nu\left(\varepsilon\left(k_{x}^{\prime \prime}, k_{y}\right)\right) d x^{\prime \prime}\right] d x^{\prime},
$$

where $x^{\prime}=p k_{x}^{\prime}$, etc, and

$$
\tilde{G}(\varepsilon)=\gamma_{i n}^{-1} f^{(0)}(\varepsilon)+\frac{\hbar}{p e F} h(\varepsilon) .
$$

The distribution function is then given by

$$
f(\mathbf{k})=\int_{0}^{\infty} \tilde{G}\left(\varepsilon\left(k_{x}+k_{x}^{\prime}, k_{y}\right)\right) \exp \left[-\frac{\hbar}{p e F} \int_{0}^{x^{\prime}} \nu\left(\varepsilon\left(k_{x}+k_{x}^{\prime \prime}, k_{y}\right)\right) d x^{\prime \prime}\right] d x^{\prime}
$$

Inserting Eq. (6) into the expression of $h\left(\varepsilon_{\mathbf{k}}\right)$ in Eq. (5), we find

$$
\tilde{G}(\varepsilon)=\frac{f^{(0)}(\varepsilon)}{\gamma_{i n}}+\frac{2}{\gamma_{e \mu} \rho(\mu)} \sum_{\mathbf{k}} \delta\left(\varepsilon-\varepsilon_{\mathbf{k}}\right)
$$




$$
\times \int_{0}^{\infty} \tilde{G}\left(\varepsilon_{n}\left(k_{x}+k_{x}^{\prime}, k_{y}\right)\right) \exp \left[-\frac{\hbar}{p e F} \int_{0}^{x^{\prime}} \nu\left(\varepsilon_{n}\left(k_{x}+k_{x}^{\prime \prime}, k_{y}\right)\right) d x^{\prime \prime}\right] d x^{\prime},
$$

where $\mu$ is the chemical potential, $\gamma_{e \mu}=p e F / \hbar \nu_{e \mu}$, and $\nu_{e \mu}=\nu_{e l}(\mu)$. The current density equals $j_{2 D}=-(e p \Delta / \hbar S) \sum_{\mathbf{k}} \sin (x) f(\mathbf{k})$, where $S$ is the area of the sample.

We solve for $\tilde{G}(\varepsilon)$ in Eq. (7) by iteration, starting from the initial value given by the first term on the right hand side. It is convenient to use $F^{*}=\hbar \nu_{\mu} / e p$ with $\nu_{\mu} \equiv \nu_{i n}+\nu_{e \mu}$ as the unit of the field. This quantity equals, for example, $F^{*} \simeq 26.3 \mathrm{Vcm}^{-1}$ for $p=25 \mathrm{~nm}$ and $\nu_{\mu}=10^{11} \sec ^{-1}$. We assume $\Delta=1 \mathrm{meV}$.

The current is shown as a function of the reduced field in Fig. 1 at the temperature $k_{B} T / \Delta \equiv T / T_{\Delta}=0.1$ for (a) five different values of $R=\nu_{i n} / \nu_{e \mu}$ for the 2D electron density $N_{2 D} / N_{\Delta}=1$ and (b) three different densities $N_{2 D} / N_{\Delta}=0.1$ (dashed curves), $N_{2 D} / N_{\Delta}=1$ (dashed-dotted curves), and $N_{2 D} / N_{\Delta}=1.7$ (solid curves). Here, $N_{\Delta}=4 \sqrt{2 m^{*} \Delta} /\left(\pi^{2} \hbar p\right)$ is the $2 \mathrm{D}$ density required to fill the band to the kink of the density of states at $\varepsilon=\Delta$ shown in the inset in (a) in units of $\rho_{0}=S \sqrt{2 m^{*}} /\left(\pi^{2} \hbar p \sqrt{\Delta}\right)$ with a level damping of $\Gamma=0.01 \Delta$ employed to avoid sharp features at the van Hove singularities. This quantity equals, for example, $N_{\Delta}=3.4 \sqrt{\Delta} \times 10^{10} \mathrm{~cm}^{-2}$ for $m^{*}=0.067$ and $p=25 \mathrm{~nm}$ with $\Delta$ in mev. The density of states has an asymptotic behavior $\rho / \rho_{0}=\pi \sqrt{\Delta / \varepsilon}$ for $\varepsilon \gg \Delta$.

The current in Fig. 1 rises, reaches a peak, and decreases monotonically as a function of the field. A similar behavior is seen at higher temperatures as will be shown later. It is seen in (a) that the current density depends significantly on the ratio $R$ in a striking contrast with the weak modulation-multiband case treated earlier. ${ }^{5}$ It is to be noted here that the current increases rapidly with $R$ but tends to saturate as $R$ becomes large. In (b), the thick (thin) curves are for $R=\nu_{i n} / \nu_{e \mu}=1(R=0.1)$. The current is larger for a larger $R$, indicating that elastic scattering is more efficient in dissipating momentum. The peak current $I_{p k}$ and the field $F_{p k}$ at the peak current depend strongly on the carrier density. The quantity $I_{p k}$ $\left(F_{p k}\right)$ has a maximum (minimum) when the chemical potential lies at the peak of the density of states in Fig. 1 corresponding to the density $N_{2 D} / N_{\Delta}=1$. The current decays as $\propto 1 / F$ asymptotically. The dependence of $I_{p k}$ (solid curves, left axis) and $F_{p k}$ (dashed curves, right 
axis) on the density is displayed in Fig. 2 for $R=1$ (thick curves) and $R=0.1$ (thin curves) at $T / T_{\Delta}=0.1$. The rough edges in the dashed curves are due to the coarseness of the scale of the reduced density employed, for convenience, for $N_{2 D} / N_{\Delta}>1$.

In the following, we investigate the temperature dependence of the current by comparing it with the current at the low temperature $T_{0} / T_{\Delta}=0.1$ studied in Figs. 1 and 2. For this purpose, we assume a linear temperature dependence for the inelastic scattering rate from electron-acoustic-phonon interaction $\nu_{i n}(T)=\nu_{i n}\left(T_{0}\right) \times T / T_{0}$. This dependence arises from the fact that the Brillouin zone size $k_{p}=\pi / p$ is very small for a superlattice, yielding a typical small phonon energy $\hbar \omega\left(k_{p}\right)<k_{B} T_{0}$ for the momentum transfer. While our figures to be shown are extended to high temperatures, it should be cautioned that the linear temperature dependence may not hold in the high-temperature regime due to additional scattering by optical phonons. Also, elastic scattering may depend on the temperature at high temperatures. We then employ the following relations for the numerical analysis for the temperature dependent parameters: $R \equiv R(T)=R_{0} \times T / T_{0}$ and $F^{*}(T)=F_{0}^{*}[R(T)+$ $1] /\left(R_{0}+1\right)$, where $R_{0}=R\left(T_{0}\right)$ and $F_{0}^{*}=F^{*}\left(T_{0}\right)$.

In Fig. 3, we show the current as a function of the field for the reduced density $N / N_{\Delta}=$ 0.7 at three temperatures $T / T_{\Delta}=0.1$ (solid curves), $T / T_{\Delta}=0.5$ (dashed curves), and $T / T_{\Delta}=1.0$ (dashed-dotted curves) for $R_{0}=0.1$ (thin black curves) and $R_{0}=5.0$ (thick red curves). The field is in units of $F_{0}^{*}$ defined at the initial temperature $T_{0} / T_{\Delta}=0.1$. The current increases monotonically as a function of the field, reaches a peak and decreases above the peak field, displaying a negative differential conductance as in Fig. 2. Roughly speaking, the current decreases (increases) as a function of the temperature for a given $R$ at a fixed field at low (high) fields. ${ }^{12}$ This behavior arises from the positive (negative) slope at low (high) fields in the field-dependent behavior in Fig. 1. Here, the quantity $F^{*}$ in the $x$-axis increases as a function of the temperature, which reduces the reduced field $F / F^{*}$, thus decreasing (increasing) the current at low (high) fields. This argument is not totally accurate for small values of $R$ (i.e., $R=0.1$ ) because $R$ increases with the rising temperature and increases the current as shown in Fig. 1 (b). However, according to (b), 
the current becomes insensitive to $R$ for large $R$. As a result, the argument presented above becomes more accurate. It should be remarked also that the current can also depend on the temperature through the distribution function.

Figure 4 displays the current density as a function of the reduced temperature for the reduced density $N_{2 D} / N_{\Delta}=0.7$ for $R_{0}=0.1$ (solid curves), $R_{0}=1.0$ (dashed curves), and $R_{0}=5.0$ (dashed-dotted curves) for the fields $F_{0}=0.5$ (thin blue curves), $F_{0}=1.0$ (thick black curves), and $F_{0}=2.0$ (thickest red curves). The fields are in units of $F_{0}^{*}$ defined at the initial temperature $T_{0}=0.1 \Delta$. The current shows a peak and decreases monotonically at high temperatures. A similar temperature-dependent behavior is displayed at a higher density $N_{2 D} / N_{\Delta}=1.7$. In this case, the current is somewhat smaller in view of the smaller density of states at the Fermi level. The rise (fall) of the curves at low (high) temperatures is sharper (slower) for larger (smaller) initial values of $R_{0}$.

In summary, we have studied the Bloch oscillations of a 2DEG at low temperatures, assuming a strong unidiretional periodic potential-modulation along the field with miniband transport and a free motion in the transverse direction. The dependence of the current on the field, the electron density, and the temperature was investigated by using a relaxationtime approximation for inelastic scattering and treating elastic scattering microscopically. For a fixed total scattering rate, the field dependence of the current was found to be sensitive to the ratio of the elastic and inelastic scattering rates in contrast with the recent result of a multiband but otherwise similar model with a weak potential modulation.

Surface superlattices considered here offer advantages such as easy device fabrication over 3D vertical superlattices and can be used for generating or detecting tunable terahertz radiation. It is also known that electron-optical phonon scattering, one of the limiting mechanisms in achieving $\mathrm{BO}$ in the terahertz range in $3 \mathrm{D}$ vertical superlattices, can be suppressed in a $2 \mathrm{D}$ lattice. ${ }^{15}$ The electric field domains formed in $3 \mathrm{D}$ superlattices have not been obsered in surface superlattices. 


\section{ACKNOWLEDGMENTS}

This work was supported by the U.S. DOE at Sandia National Laboratories under

Contract No.DE-AC04-94AL85000. Computational resources were provided by the DOE NERSC facility. 


\section{REFERENCES}

${ }^{1}$ F. Bloch, Z. Phys. 52, 555 (1928).

${ }^{2}$ L. Esaki and R. Tsu, IBM J. Res. Dev. 14, 61 (1970).

${ }^{3}$ R. R. Gerhardts, Phys. Rev. B 48, 9178 (1993).

${ }^{4}$ S. K. Lyo, Phys. Rev. B 77, 195306 (2008).

${ }^{5}$ S. K. Lyo and W. Pan, Phys. Rev. B 84, 195320 (2011).

${ }^{6}$ A. Sibille, J. F. Palmier, H. Wang, and F. Mollot, Phys. Rev. Lett. 64, 52 (1990).

${ }^{7}$ H. T. Grahn, K. von Klitzing, K. Ploog, and G. H. Dhler,Phys. Rev. B 43, 12094 (1991).

${ }^{8}$ N. Sekine and K. Hirakawa, Phys. Rev. Lett. 94, 057408 (2005).

${ }^{9}$ F. G. Bass and E. A. Rubinshtein, Fiz. Tverd. Tela Leningrad 19, 1379 (1977),[Sov. Phys. Solid State 19, 800 (1977).]

${ }^{10}$ R. A. Suris and B. S. Shchamkhalova, Fiz. Tekh. Poluprovodn. S.-Peterburg 18, 1178 (1984) [Sov. Phys. Semicond. 18, 738 (1984).]

11 A. A. Ignatov, K. F. Renk, and E. P. Dodin, Phys. Rev. Lett. 70, 1996 (1993).

${ }^{12}$ Full references to past works are given in Ref. $^{5}$ and $^{4}$

${ }^{13}$ W. Pan, S. K. Lyo, J. L. Reno, J. A. Simmons, D. Li and S. R. J. Brueck, Appl. Phys. Lett. 92, 052104 (2008).

${ }^{14}$ For $R=5$ (thick curves), the high-field behavior is seen much above the field range shown in this figure, where the dashed curve falls eventually below the dashed-dotted curve.

${ }^{15}$ I. A. Dmitriev and R. A. Suris, Semiconductors 35, 212 (2001). 


\section{FIGURES}

FIG. 1. The current density as a function of the reduced field at the temperature $k_{B} T / \Delta=0.1$ for (a) several $R$ values for $N_{2 D} / N_{\Delta}=1$ and (b) $N_{2 D} / N_{\Delta}=0.1$ (dashed curves), $N / N_{\Delta}=1$ (dashed-dotted curves), and $N / N_{\Delta}=1.7$ (solid curves) for $R=1$ (thick curves) and $R=0.1$ (thin curves). The inset in (a) shows the density of states. The quantities $F^{*}$ and $N_{\Delta}$ are defined in the text.

FIG. 2. The peak current density $I_{p k}$ (solid curves, left axis) and the field $F_{p k}$ at the peak current (dashed curves, right axis) as a function of the $2 \mathrm{D}$ electron density for the I-V curves in Fig. 1 at $k_{B} T / \Delta=0.1$. The thick (thin) curves are for $R=1(R=0.1)$. A fine (coarse) grading is taken for $N_{2 D} / N_{\Delta} \leq 1\left(N_{2 D} / N_{\Delta}>1\right)$. The quantities $F^{*}$ and $N_{\Delta}$ are defined in the text.

FIG. 3. The current density as a function of the field for $N_{2 D} / N_{\Delta}=0.7$ at $T / T_{\Delta}=0.1$ (solid curves), $T / T_{\Delta}=0.5$ (dashed curves), and $T / T_{\Delta}=1.0$ (dashed-dotted curves) for $R_{0}=0.1$ (thin black curves) and $R_{0}=5.0$ (thick red curves). The thick (thin) curves are for $R_{0}=5\left(R_{0}=0.1\right)$. The quantities $N_{\Delta}, R_{0}$, and $T_{\Delta}$ are defined in the text.

FIG. 4. The current density as a function of $T / T_{\Delta}$ for $N_{2 D} / N_{\Delta}=0.7$ for $R_{0}=0.1$ (solid curves), $R_{0}=1.0$ (dashed curves), and $R_{0}=5.0$ (dashed-dotted curves) for $F / F_{0}^{*}=0.5$ (thin blue curves), $F / F_{0}^{*}=1.0$ (thick black curves), and $F / F_{0}^{*}=2.0$ (thickest red curves). The quantities $N_{\Delta}, R_{0}, F_{0}^{*}$, and $T_{\Delta}$ are defined in the text. 

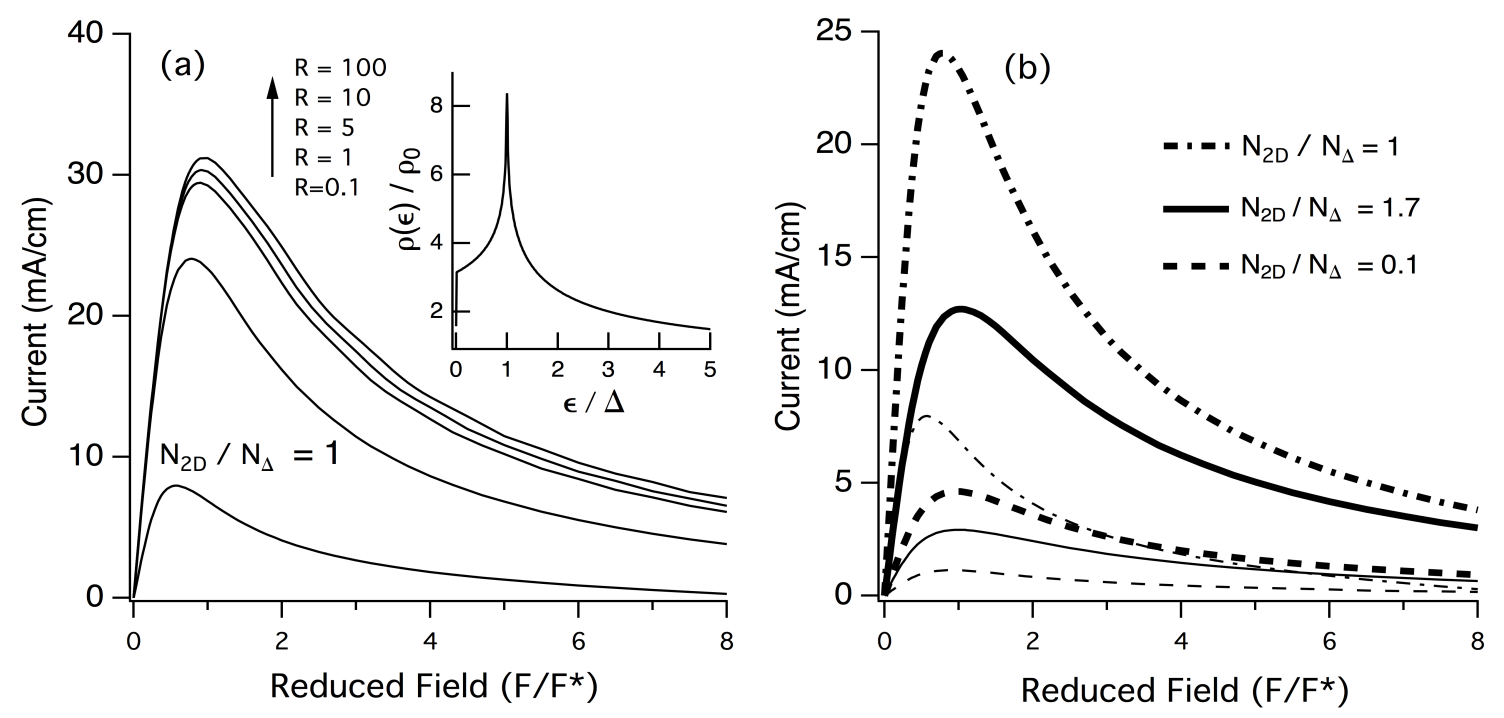

Fig. 1 


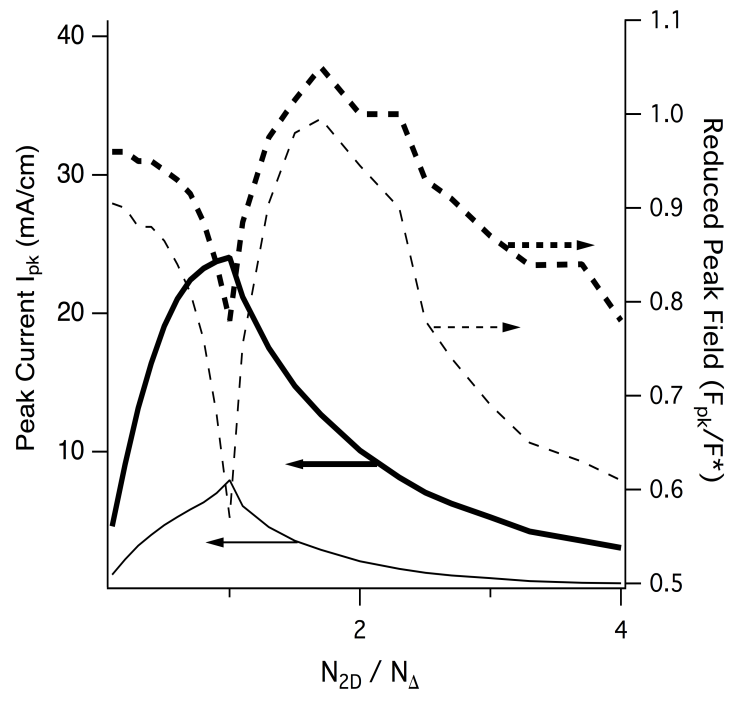

Fig. 2 


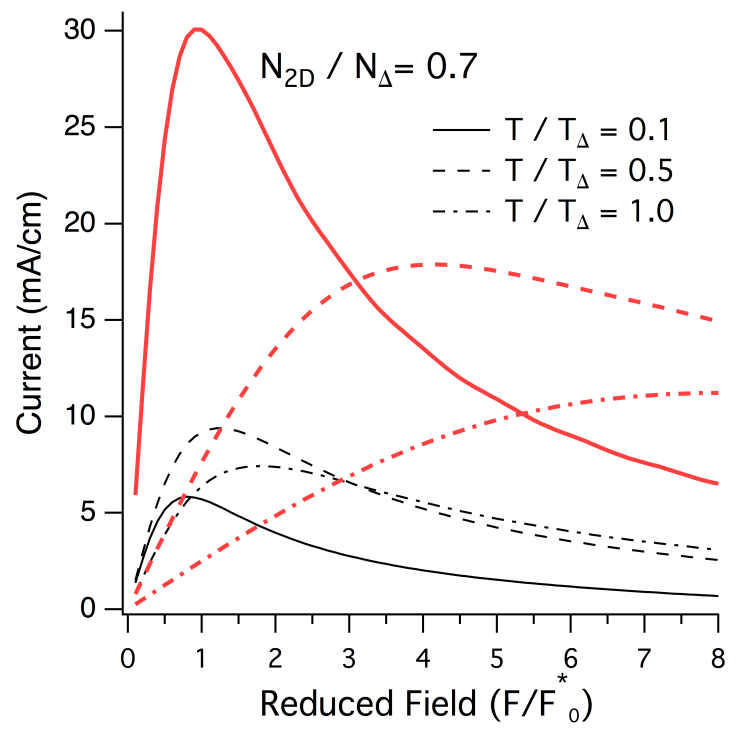

Fig. 3 


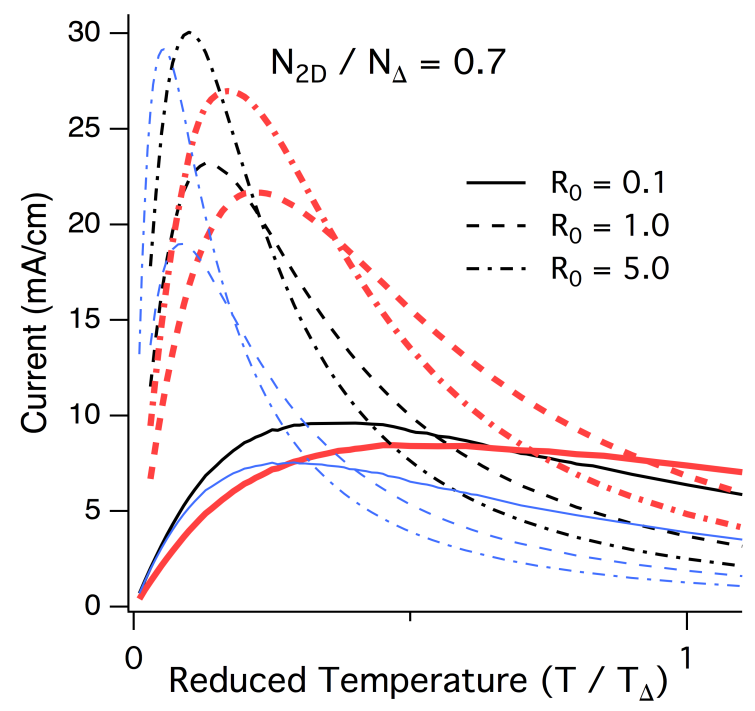

Fig.4 